(c) American Dairy Science Association, 2004.

\title{
Aroma Compounds in Sweet Whey Powder
}

\author{
S. S. Mahajan, L. Goddik, and M. C. Qian \\ Department of Food Science and Technology \\ Oregon State University, Corvallis 97331
}

\section{ABSTRACT}

Aroma compounds in sweet whey powder were investigated in this study. Volatiles were isolated by solvent extraction followed by solvent-assisted flavor evaporation. Fractionation was used to separate acidic from nonacidic volatiles. Gas chromatography/mass spectrometry and gas chromatography/olfactometry were used for the identification of aroma compounds. Osme methodology was applied to assess the relative importance of each aroma compound. The most aroma-intense free fatty acids detected were acetic, propanoic, butanoic, hexanoic, heptanoic, octanoic, decanoic, dodecanoic, and 9-decenoic acids. The most aroma-intense nonacidic compounds detected were hexanal, heptanal, nonanal, phenylacetaldehyde, 1-octen-3-one, methional, 2,6-dimethylpyrazine, 2,5-dimethylpyrazine, 2,3dimethylpyrazine, 2,3,5-trimethylpyrazine, furfuryl alcohol, p-cresol, 2-acetylpyrrole, maltol, furaneol, and several lactones. This study suggested that the aroma of whey powder could comprise compounds originating from milk, compounds generated by the starter culture during cheese making, and compounds formed during the manufacturing process of whey powder.

(Key words: sweet whey powder, aroma, Osme, pyrazine)

Abbreviation key: GCMS = gas chromatography/ mass spectrometry, GCO = gas chromatography/olfactometry, $\mathbf{R I}=$ retention index.

\section{INTRODUCTION}

Sweet whey powder is obtained by drying fluid whey collected from the manufacture of Cheddar, Swiss, Mozzarella, Monterey Jack, and similar cheeses. It typically contains about $70 \%$ lactose, $1.5 \%$ fat, $12 \%$ proteins, $4 \%$ moisture, and 8.5\% other solids (U.S. Dairy Export Council, 2004). Whey has become an important ingredient in dairy and other food products (Whetstine et al.,

Received April 19, 2004.

Accepted August 22, 2004.

Corresponding author: M. C. Qian; e-mail: michael.qian@ oregonstate.edu.
2003) with a total production greater than 1.3 billion pounds in 2003 (USDA, 2004). In spite of its large production, whey is often thought of as having an undesirable flavor that is unpleasant to consumers, which limits its use in bland or delicately flavored foods (Whetstine et al., 2003).

Flavor quality in liquid whey has been evaluated recently. Karagul-Yuceer et al. (2003) identified aroma compounds in liquid Cheddar whey and found that 2,3butanedione, hexanal, 2-acetyl-1-pyrroline, methional, (E,E)-2,4-nonadienal, (E,E)-2,4-decadienal, and various short-chain volatile acids could be important to the aroma of liquid whey. Whetstine et al. (2003) found that whey made from different types of cheeses has different flavors, and that flavor variability exists within the same cheese type. Tomaino et al. (2004) studied the flavor variability of liquid whey produced from laboratory-scale Cheddar cheese using individual strains of Lactococcus lactis spp. lactis. They confirmed that there were differences in liquid whey produced in a noncommercial setting from different starter culture strains.

Whey powder is expected to have a different flavor profile than liquid whey because of the further steps involved in its processing such as concentration and spray drying. These additional steps may reduce or generate flavor compounds, thus change the flavor profile of whey powder. Although Ferretti and Flanagan (1971) reported a wide variety of volatiles in whey powder, no direct information was provided about its flavor components. Livney and Bradley (1994) reported saltiness, brothiness, diacetyl, bitterness, and acidity as some of the flavor characteristics in acid whey. They suggested that whey flavor may be due to components traced to the original milk or developed during processing, but they did not identify those aroma compounds. Whitfield (1992) hypothesized that the Maillard reaction and lipid oxidation initiate flavor development in whey products but so far, there is no experimental data to support this hypothesis.

To date, no information is available on the aroma compounds present in sweet whey powder and hence this study is aimed at identification of flavor compounds in this dairy ingredient. In this research, flavor compounds in commercially produced sweet whey powder 
were analyzed using gas chromatography/olfactometry (GCO) and gas chromatography/mass spectrometry (GCMS).

\section{MATERIALS AND METHODS}

\section{Sweet Whey Powder}

Fresh sweet whey powder samples (Cheddar cheese whey, US extra grade) obtained from the Midwest (sample A) and Pacific Northwest (sample B) were used for analysis. The samples were stored frozen $\left(-37^{\circ} \mathrm{C}\right)$ and used within 3 mo.

\section{Aroma Extraction}

One kilogram of sweet whey powder was mixed with $500 \mathrm{~mL}$ of 2:1 freshly distilled pentane (Mallinckrodt Baker, Phillipsburg, NJ) and diethyl ether (Honeywell International, Muskegon, MI) for $4 \mathrm{hr}$ during each extraction, in a tightly capped Erlenmeyer flask with intermittent shaking every $15 \mathrm{~min}$. Extractions were repeated 3 times. All extracts were combined and concentrated to $500 \mathrm{~mL}$ under the hood. The extracts were distilled at $50^{\circ} \mathrm{C}$ by solvent-assisted flavor evaporation under vacuum (28 inch $\mathrm{Hg}$ ) to separate volatiles from nonvolatiles. Liquid nitrogen was used for condensation of volatiles. The distillate was further concentrated to $50 \mathrm{~mL}$ by letting it evaporate in a fume hood, and was then subjected to fractionation.

\section{Fractionation}

The aroma concentrate was separated into acidic and nonacidic (neutral, basic) fractions. The concentrate (50 $\mathrm{mL}$ ) was washed twice with $3 \mathrm{~mL}$ of $1 M$ sodium bicarbonate solution containing sodium chloride (Sigma Chemical Co., St. Louis, MO). The upper (ether) phase, containing the nonacidic volatiles, was dried over anhydrous sodium sulfate (EMP Chemicals, Gibbstown, NJ) and concentrated to $500 \mu \mathrm{L}$ for sample A and to $50 \mu \mathrm{L}$ for sample B under a stream of nitrogen. The pooled aqueous phase (bottom layer) was acidified to $\mathrm{pH} 2.5$ with $\mathrm{HCl}$ (Reagent grade, USP, Integra Chemical Company, Renton, WA). The acidic volatiles were further extracted (3×) with $0.5 \mathrm{~mL}$ of freshly distilled diethyl ether. The diethyl ether extract was then dried over anhydrous sodium sulfate and was concentrated to 500 $\mu \mathrm{L}$ for sample A and to $50 \mu \mathrm{L}$ for sample B with a stream of nitrogen.

\section{Gas Chromatography}

A Hewlett Packard 5890 gas chromatograph equipped with a flame ionization detector and an olfac- tometer was used. Samples were analyzed on a DB-Wax column $(30 \mathrm{~m} \times 0.25 \mathrm{~mm}$ i.d. coated with crosslinked polyethylene glycol $20 \mathrm{M}$, film thickness, $0.5 \mu \mathrm{m}$; J\&W Scientific, Folsom, CA) and a DB-5 column (30 m $\times$ $0.32 \mathrm{~mm}$ i.d. coated with crosslinked $5 \%$ phenyl-methyl polysiloxane, film thickness, $1 \mu \mathrm{m}$; J\&W Scientific). The column effluent was split 1:1 (by volume) into the flame ionization detector and a heated sniffing port with a splitter (Alltech Associates, Inc., Deerfield, IL). Gas chromatography operating parameters were: injection port temperature $=250^{\circ} \mathrm{C}$, detector temperature $=$ $250^{\circ} \mathrm{C}$, oven temperature from 40 to $230^{\circ} \mathrm{C}$ at the rate of $5^{\circ} \mathrm{C} / \mathrm{min}$ with initial and final hold times of 2 and 10 min for DB-Wax, and 4 and 10 min for DB-5 columns, respectively. The helium column flow rate was $2.0 \mathrm{~mL} /$ min measured at $25^{\circ} \mathrm{C}$. A sample $(2 \mu \mathrm{L})$ of acidic fraction was injected onto the DB-Wax column and $2 \mu \mathrm{L}$ of nonacidic fraction was injected onto both columns at a split ratio of 1:1.

\section{GCO}

Seven volunteers from Oregon State University performed GCO analysis on the whey samples. Panelists participated in 4 trial runs of each acidic and nonacidic fraction of the 2 samples to identify product attributes, clarify reference terminology, and verify or establish references for flavor and intensity values (Da Silva et al., 1994). Each fraction was used at the same concentration as required for analysis. At the end of 4 sessions, 4 subjects were chosen based upon their ability to consistently perceive and describe odors as the aroma compounds eluted from the sniff port. During actual analysis, sniffing was performed twice on each fraction by each panelist using the Osme technique (McDaniel et al., 1990) with minor modification. A 16-point intensity scale, with $0=$ none, and $15=$ extreme, was used to assign intensity to aromas detected (Miranda-Lopez et al., 1992; Roberts et al., 2003).

\section{GCMS}

Gas chromatography/mass spectrometry analysis was performed using an Agilent 6890 gas chromatograph equipped with a 5973 mass selective detector. System software control and data analysis were performed using enhanced ChemStation Software, G1707CA v. C.00.01.08 (Agilent Technologies, Inc., Wilmington, DE). Volatile separation was achieved with 2 fused silica capillary columns: DB-Wax $(30 \mathrm{~m} \times 0.25$ $\mathrm{mm}$ i.d. coated with crosslinked polyethylene glycol $20 \mathrm{M}$, film thickness, $0.5 \mu \mathrm{m}$, J\&W Scientific) and DB$5(30 \mathrm{~m} \times 0.32 \mathrm{~mm}$ i.d. coated with crosslinked $5 \%$ phenyl-methyl polysiloxane, film thickness, $1 \mu \mathrm{m}$, J\& 
W Scientific). The oven temperature was programmed as for the GCO analysis. Injector, detector transfer line, and ion source temperatures were 250,280 , and $230^{\circ} \mathrm{C}$ respectively. Helium was used as carrier gas with constant flow of $2 \mathrm{~mL} / \mathrm{min}$. A splitter was used at the end the column as described by Qian and Reineccius (2002). One milliliter of the flow was directed to mass spectrometry and the remainder was directed to an olfactometer. Standard electron impact mass spectroscopy was used, and the ionization voltage was set at $70 \mathrm{eV}$. An $\mathrm{m} / z$ range of 35 to 300 was collected at $5.27 \mathrm{scans} / \mathrm{s}$. A sample $(2 \mu \mathrm{L})$ of each fraction was injected in the splitless mode.

\section{Retention Index}

A sample containing series of hydrocarbons $\left(\mathrm{C}_{5}\right.$ to $\mathrm{C}_{25}$ ) was injected on the DB-Wax and DB-5 columns using the same conditions mentioned as above for the 2 samples. Retention indices (RI) for the aroma compounds were calculated using the modified Kovats method (Van Den Dool and Kratz, 1963).

\section{Identification of Odorants}

Positive identifications were made by comparing calculated RI with standards, panelist's aroma description, and individual mass spectra (Wiley 275.L mass spectral database; Agilent Technologies, Inc.). Tentative identifications were based on retention indices and odor descriptors.

\section{RESULTS AND DISCUSSION}

Many acids were identified on the DB-Wax column in sweet whey powder samples from the Midwest (sample A) and Pacific Northwest (sample B). The most important acids found in sample A were acetic, butanoic, 3-methylbutanoic, hexanoic, and octanoic acids based on their high Osme value (Osme value >8) (Table 1). Acetic acid, with its sharp vinegar-like odor, could be one of the most prominent among the identified acids. It showed a high Osme value indicating its important role in imparting the unique aroma to sweet whey powder. Butanoic, hexanoic, and octanoic acids contributed to the cheesy and rancid odors. Of these, butanoic acid showed high aroma intensity. 3-Methylbutanoic acid gave a sweaty odor with a relatively high Osme value. Pentanoic and dodecanoic acids gave pungent odors, and octanoic acid gave an animal-like odor. Formic acid with its pungent odor showed a weak intensity in both samples. Propanoic, 2-methylpropanoic, decanoic, and dodecanoic acids showed relatively low Osme values in the 2 samples. 9-Decenoic acid gave a fatty aroma with weak Osme values in both samples. Among the 12 acids identified in the acidic fraction, all were present in sample A, whereas 2-methylpropanoic and dodecanoic acids were absent in sample B. Short-chain FFA have been identified in many foods including cheese (Aston and Dulley, 1982; Qian and Reineccius, 2002; Qian et al., 2002) and liquid whey (Karagul-Yuceer et al., 2003; Whetstine et al., 2003; Tomaino et al., 2004). Free fatty acids can be formed through the action of milk or bacterial lipases on triglycerides and phospholipids (Ferretti and Flanagan, 1971; Badings, 1991; Kim et al., 2003). Because the FFA were isolated by their chemical properties, and well separated and identified on the DBWax column, analysis was not necessary on an additional column.

The nonacidic fraction was analyzed on DB-Wax (Table 2)and DB-5 (Table 3) columns. The chemical classes of compounds identified on the 2 columns consisted of aldehydes, ketones, lactones, sulfur-containing compounds, and many others.

Many aldehydes were identified in sample A to have very intense aroma on DB-Wax column (Table 2). Saturated aldehydes identified were hexanal, heptanal, and nonanal, and they had characteristic grassy, green, floral aromas. Most of the unsaturated aldehydes were tentatively identified by their RI and descriptive odor properties. The most prominent of these were (E)-2octenal, (Z)-2-nonenal, (E,Z)-2,6-nonadienal, (E,E)-2,4nonadienal, (E,E)-2,4-octadienal, (E,Z)-2,4-decadienal and (E,E)-2,4-decadienal. These aldehydes are formed from the autooxidation of unsaturated fatty acids (Wadodkar et al., 2002). Phenylacetaldehyde, an aroma compound with a floral note, was detected only in sample A. This compound is believed to have derived from Strecker degradation of phenylalanine (Counet et al, 2002).

Two straight chain ketones were identified in the whey powder. Diacetyl was identified with its characteristic buttery note. It is a product of citrate metabolism, involving Lactococcus lactis ssp. lactis biovar diacetylactis during cheese making (Kaneko et al., 1987; Bendall, 2001; Kieronczyk et al., 2003). It is believed that diacetyl undergoes reduction to form acetoin, an aromatic hydroxy ketone (Hugenholtz et al., 2000), which was also detected with its typical butter-like aroma in both samples. 1-octen-3-one was detected with a typical mushroom-like odor. Although the intensity for this compound was from moderate to small on DBWax (Table 2) and moderate on DB-5 (Table 3), it showed unique earthy aroma characteristics to the odor profile of whey powder.

Several sulfur-containing compounds were identified on the DB-Wax column (Table 2). Both dimethyl disulfide and dimethyl trisulfide have an onion, cabbage 
Table 1. Aroma compounds in the acidic fraction of sweet whey powder analyzed on DB-Wax column.

\begin{tabular}{|c|c|c|c|c|c|}
\hline \multirow[b]{2}{*}{ Compound } & \multirow[b]{2}{*}{$\mathrm{RI}^{1}$} & \multirow[b]{2}{*}{ Aroma } & \multicolumn{2}{|c|}{ Osme value } & \multirow[b]{2}{*}{ Identification $^{2}$} \\
\hline & & & $\mathrm{A}$ & B & \\
\hline Acetic acid & 1460 & Vinegar-like & 11 & 9 & $1,2,3$ \\
\hline Formic acid & 1510 & Pungent & 5 & 6 & $1,2,3$ \\
\hline Propanoic acid & 1550 & Rancid & 5 & 2 & $1,2,3$ \\
\hline 2-Methylpropanoic acid & 1580 & Buttery & 6 & 0 & $1,2,3$ \\
\hline Butanoic acid & 1645 & Cheesy & 12 & 10 & $1,2,3$ \\
\hline 3-Methylbutanoic acid & 1682 & Sweaty & 9 & 8 & $1,2,3$ \\
\hline Pentanoic acid & 1755 & Pungent & 6 & 4 & $1,2,3$ \\
\hline Hexanoic acid & 1858 & Rancid & 11 & 6 & $1,2,3$ \\
\hline Heptanoic acid & 1971 & Cheesy & 6 & 7 & $1,2,3$ \\
\hline Octanoic acid & 2072 & Animal-like & 9 & 6 & $1,2,3$ \\
\hline Decanoic acid & 2294 & Soapy & 6 & 4 & $1,2,3$ \\
\hline 9-Decenoic acid & 2348 & Fatty & 4 & 2 & $1,2,3$ \\
\hline Dodecanoic acid & 2505 & Soapy & 5 & 0 & $1,2,3$ \\
\hline
\end{tabular}

note, while methional has a cooked potato aroma. Methional could be a very important flavor compound because of its high Osme value in sample A. It was detected very close to the elution of 2,6-dimethylpyrazine, but was differentiated by the panelists with a cooked potato note for methional and a cooked meaty note for 2,6-dimethylpyrazine. Dimethyl sulfide, dimethyl trisulfide, and methional have been identified in cheese (Aston and Dulley, 1982; Qian et al., 2002, Boscaini et al., 2003; Qian and Reineccius, 2003) and liquid whey (Karagul-Yuceer et al., 2003; Whetstine et al., 2003; Tomaino et al., 2004). Degradation of methionine can form dimethyl sulfide and methanethiol; and methanethiol can be further oxidized to form dimethyl disulfide and dimethyl trisulfide (Bendall, 2001). Dimethyl sulfide can be oxidized to dimethyl sulfone (Livney and Bradley, 1994). Although a peak was detected for dimethyl sulfone by mass spectroscopy, no aroma was sensed. Methional is thought to be generated from Strecker degradation of methionine (Aston and Dulley, 1982; Da Silva et al., 1993; Fu et al., 2002; Boscaini et al., 2003).

Pyrazines are nitrogen-containing heterocyclic compounds which are recognized for their characteristic baked and roasted potato, nut, and meat-like aromas. The most intense of these were 2,6-dimethylpyrazine, 2,5-dimethylpyrazine, 2,3-dimethylpyrazine, 2,3,5-trimethylpyrazine for sample A (Table 2). Most of these pyrazines had moderate Osme values in sample B except 2,5-dimethylpyrazine (Osme value of 10). 2,6-Dimethylpyrazine was only identified in sample A, whereas 2-ethylpyrazine was only identified in sample B. Most of these pyrazines have been identified in Parmesan cheese as compounds responsible for the nutty aroma (Qian and Reineccius, 2002). 2-Methoxy-3-isopropylpyrazine and 2-isobutyl-3-methoxypyrazine have been identified in liquid whey (Karagul-Yuceer et al., 2003), but were not identified in this study. Pyrazines are the products of Maillard reaction (Friedman, 1996; Alasalvar et al., 2003), possibly formed during concentration, spray drying, or other processes involved in the manufacturing of whey powder.

2-Acetylpyrrole with its characteristic medicinal odor was seen only in sample A (Table 2). 2-Acetylthiazole, 2-acetyl-1-pyrroline, and 2-propionyl-1-pyrroline were tentatively identified to have roasted nuts and fried kind of aromas. 2-Acetyl-1-pyrroline is believed to be formed by the interaction of pyruvaldehyde with 1-pyrroline, which is formed from proline (Bendall, 2001; Karagul-Yuceer et al., 2001). 2-Acetyl-1-pyrroline and 2-propionyl-1-pyrroline possess very low odor thresholds and are frequently cited as compounds that directly contribute to roasted or smoky flavors (Da Silva et al., 1993; Karagul-Yuceer et al., 2003).

Maltol and furaneol were identified in the whey powder that gave moderate to large intensity in both samples with a burnt sugar-like aroma for maltol and a sweet aroma for furaneol (Table 2). Maltol and furaneol are believed to be products of sugar degradation ( $\mathrm{Da}$ Silva et al., 1993; Wadodkar et al., 2002). Sotolon gave a spicy note, with high intensity on both samples on DB-Wax column (Table 2). This compound is thought to be formed from $\alpha$-ketobutyric acid and acetaldehyde (Takahashi et al., 1976). Maltol and sotolon have been identified in liquid whey (Karagul-Yuceer et al., 2003), and furaneol was found in stored nonfat dry milk (Karagul-Yuceer et al., 2001).

Most of the lactones were detected only by the DBWax column (Table 2). Only a few compounds such as delta-decalactone and gamma-hexalactone were detected by both columns. Fruity, nutty, and dairy aromas were associated with lactones in both samples, and 
Table 2. Aroma compounds in nonacidic fraction of sweet whey powder analyzed on DB-Wax column.

\begin{tabular}{|c|c|c|c|c|c|}
\hline \multirow[b]{2}{*}{ Compound $^{1}$} & \multirow[b]{2}{*}{$\mathrm{RI}^{2}$} & \multirow[b]{2}{*}{ Aroma } & \multicolumn{2}{|c|}{ Osme value } & \multirow[b]{2}{*}{ Identification } \\
\hline & & & A & B & \\
\hline Diacetyl & 1005 & Buttery & 7 & 9 & 1,2 \\
\hline Dimethyl disulfide* & 1095 & Cooked vegetable & 4 & 6 & 1,2 \\
\hline Hexanal & 1097 & Grassy & 5 & 4 & $1,2,3$ \\
\hline Heptanal & 1199 & Grassy & 12 & 10 & $1,2,3$ \\
\hline (Z)-4-Heptenal* & 1231 & Sweet & 8 & 8 & 1,2 \\
\hline 1-Octen-3-one & 1307 & Mushroom & 6 & 3 & $1,2,3$ \\
\hline Unknown* & 1323 & Buttery & 5 & 8 & 1 \\
\hline 2-Acetyl-1-pyrroline* & 1331 & Roasted nuts & 7 & 0 & 1,2 \\
\hline 2,6-Dimethylpyrazine & 1347 & Cooked meat & 15 & 0 & $1,2,3$ \\
\hline 2,5-Dimethylpyrazine & 1348 & Cooked potato & 12 & 10 & $1,2,3$ \\
\hline 2-Ethylpyrazine* & 1357 & Roasted nuts & 0 & 6 & 1,2 \\
\hline Dimethyl trisulfide* & 1367 & Sulfury, cabbage & 8 & 7 & 1,2 \\
\hline 2,3-Dimethylpyrazine & 1375 & Nutty & 10 & 7 & $1,2,3$ \\
\hline 2-Acetylthiazole* & 1397 & Roasted nuts & 9 & 10 & 1,2 \\
\hline Nonanal & 1406 & Floral & 10 & 7 & $1,2,3$ \\
\hline (E)-2-Octenal* & 1416 & Roasted peanuts & 10 & 4 & 1,2 \\
\hline 2-Propionyl-1-pyrroline* & 1434 & Fried & 12 & 13 & 1,2 \\
\hline Methional & 1464 & Cooked potato & 13 & 8 & $1,2,3$ \\
\hline (Z)-2-Nonenal* & 1493 & Fatty & 10 & 0 & 1,2 \\
\hline 2,3,5-Trimethylpyrazine & 1517 & Roasted & 9 & 5 & $1,2,3$ \\
\hline Unknown & 1522 & Pungent & 6 & 10 & 1 \\
\hline Unknown & 1545 & Sour & 7 & 12 & 1 \\
\hline (E)-2-Nonenal* & 1558 & Pungent & 8 & 13 & 1,2 \\
\hline (E,Z)-2,6-Nonadienal* & 1604 & Cucumber & 8 & 13 & 1,2 \\
\hline Furfuryl alcohol & 1613 & Burnt rubber & 7 & 5 & $1,2,3$ \\
\hline (E,E)-2,4-Octadienal* & 1615 & Cucumber & 15 & 10 & 1,2 \\
\hline Unknown & 1619 & Rancid & 7 & 0 & 1 \\
\hline Phenylacetaldehyde & 1632 & Floral & 8 & 0 & $1,2,3$ \\
\hline (E,Z)-2,4-Nonadienal* & 1646 & Green & 7 & 7 & 1,2 \\
\hline (E,E)-2,4-Nonadienal* & 1681 & Rancid & 13 & 7 & 1,2 \\
\hline Gamma hexalactone & 1736 & Coconut & 10 & 9 & $1,2,3$ \\
\hline (E,Z)-2,4-Decadienal* & 1752 & Green & 13 & 13 & 1,2 \\
\hline (E,E)-2,4-Decadienal* & 1795 & Baked & 7 & 10 & 1,2 \\
\hline Delta-octalactone & 1988 & Baked & 5 & 5 & $1,2,3$ \\
\hline 2-Acetylpyrrole & 2002 & Herbal medicine & 12 & 0 & $1,2,3$ \\
\hline Maltol & 2012 & Caramel & 8 & 12 & $1,2,3$ \\
\hline Furaneol & 2033 & Sweet & 8 & 8 & $1,2,3$ \\
\hline Unknown & 2056 & Caramel & 13 & 0 & 1 \\
\hline p-Cresol & 2103 & Cattle & 11 & 6 & $1,2,3$ \\
\hline Gamma-decalactone & 2143 & Milk powder & 9 & 10 & $1,2,3$ \\
\hline Sotolon* & 2186 & Spicy & 10 & 15 & 1,2 \\
\hline Delta-decalactone & 2220 & Fruity & 10 & 9 & $1,2,3$ \\
\hline Delta-undecalactone* & 2345 & Fruity & 4 & 14 & 1,3 \\
\hline Gamma-dodecalactone & 2384 & Cheesy & 8 & 5 & $1,2,3$ \\
\hline Delta-dodecalactone & 2466 & Buttery & 3 & 4 & $1,2,3$ \\
\hline Skatole* & 2486 & Animal-like & 6 & 8 & 1,2 \\
\hline
\end{tabular}

${ }^{1}$ Compounds with * are tentatively identified.

${ }^{2} \mathrm{RI}=$ Retention index.

${ }^{3}$ Identification: 1 = aroma descriptor; 2 = retention index $(\mathrm{RI}) ; 3$ = mass spectroscopy.

aroma intensities varied from very low to very high. Parlimnet et al. (1966) concluded that the precursors for lactones were triglycerides containing a hydroxyl acid moiety.

p-Cresol, a phenolic compound with a typical cattlelike odor contributed a high Osme value for sample A and a weak Osme value for sample B on the DB-Wax column (Table 2). It has long been known as the factor responsible for the smell of cow urine. It imparts a barn/medicine-like flavor to milk upon liberation from conjugated precursors (Bendall, 2001; Kim et al., 2003).
Skatole was also identified in this study on both columns (Tables 2 and 3). It strongly associated with an unpleasant smell of cow feces. It is produced from the amino acid L-tryptophan in the cow's rumen from the diet. Skatole has been found in liquid whey and in nonfat dry milk (Bendall, 2001; Karagul-Yuceer et al., 2001).

Similar compounds were identified in samples A and B. Sample A showed 45 aroma compounds on DB-Wax and 21 on DB-5. In addition, sample A showed 5 unknowns on DB-Wax and 1 on DB-5. Sample B showed 
Table 3. Aroma compounds in nonacidic fraction of sweet whey powder analyzed on DB-5 column.

\begin{tabular}{|c|c|c|c|c|c|}
\hline \multirow[b]{2}{*}{ Compound $^{1}$} & \multirow[b]{2}{*}{$\mathrm{RI}^{2}$} & \multirow[b]{2}{*}{ Aroma } & \multicolumn{2}{|c|}{ Osme value } & \multirow[b]{2}{*}{ Identification $^{3}$} \\
\hline & & & $\mathrm{A}$ & B & \\
\hline Diacetyl* & 606 & Buttery & 5 & 8 & 1,2 \\
\hline Hexanal & 789 & Grassy & 5 & 5 & $1,2,3$ \\
\hline Heptanal & 899 & Grassy & 9 & 0 & $1,2,3$ \\
\hline Methional & 902 & Cooked potato & 9 & 3 & $1,2,3$ \\
\hline 2,6-Dimethylpyrazine & 906 & Cooked meat & 11 & 0 & $1,2,3$ \\
\hline 2-Acetyl-1-pyrroline* & 916 & Roasted nuts & 11 & 3 & 1,2 \\
\hline 1-Octen-3-one & 974 & Mushroom & 6 & 8 & $1,2,3$ \\
\hline Gamma-hexalactone & 1047 & Coconut & 10 & 8 & $1,2,3$ \\
\hline 2-Acetylpyrrole & 1072 & Herbal medicine & 8 & 0 & $1,2,3$ \\
\hline p-Cresol & 1074 & Cattle & 11 & 3 & $1,2,3$ \\
\hline Maltol & 1088 & Burnt sugar & 10 & 12 & $1,2,3$ \\
\hline Nonanal & 1101 & Floral & 6 & 0 & $1,2,3$ \\
\hline Sotolon* & 1108 & Spicy & 11 & 0 & 1,2 \\
\hline (Z)-2-Nonenal* & 1147 & Fatty & 8 & 6 & 1,2 \\
\hline (E,Z)-2,6-Nonadienal* & 1153 & Cucumber & 8 & 12 & 1,2 \\
\hline (E)-2-Nonenal* & 1155 & Pungent & 9 & 11 & 1,2 \\
\hline (E,E)-2,4-Nonadienal* & 1211 & Rancid & 8 & 6 & 1,2 \\
\hline (E,E)-2,4-Decadienal* & 1313 & Baked & 9 & 6 & 1,2 \\
\hline Skatole* & 1391 & Animal-like & 7 & 11 & 1,2 \\
\hline Unknown & 1482 & Floral & 10 & 11 & 1 \\
\hline Delta-decalactone & 1491 & Fruity & 12 & 0 & $1,2,3$ \\
\hline
\end{tabular}

39 aroma compounds on DB-wax and 15 on DB-5. Most of these compounds were identified in both samples. Some compounds, however, were only identified in one sample. For example, ethylpyrazine was identified on DB-Wax column in sample B but was absent in sample A, whereas 2-acetyl-1-pyrroline, 2,6-dimethylpyrazine, (Z)-2-nonenal, phenylacetaldehyde, and 2-acetyl pyrrole were identified in sample A, but were absent in sample B. The Osme values also varied substantially. To perceive the aroma, sample B was evaluated at a concentration 10 times higher than sample A. The fewer aroma compounds identified in sample B and low aroma intensity suggested a bland, clean whey powder.

\section{CONCLUSION}

Important aroma volatiles analyzed in sweet whey powder were the short-chain fatty acids, aldehydes and ketones, lactones, sulfur compounds, phenols, indoles, pyrazines, furans, and pyrroles. Some of them are derived from milk or formed during cheese making, whereas others are formed during whey powder processing. Autooxidation of lipids, caramelization of sugar, and Maillard browning can explain the generation of many aroma compounds. Although more sensory work is needed to validate the results, it is likely that the identified compounds are responsible for the characteristic aroma of sweet whey powder.

\section{ACKNOWLEDGMENTS}

The authors would like to thank Tillamook County Creamery Association for their financial support.

\section{REFERENCES}

Acree, T. E., J. Barnard, and D. G. Cunningham. 1984. A procedure for the sensory analysis of gas chromatographic effluents. J. Food Chem. 14:273-286.

Alasalvar, C., F. Shahidi, and K. Cadwallader. 2003. Comparison of natural and roasted Turkish tombul hazelnut (Corylus avellana L.) volatiles and flavor by DHA/GC/MS and descriptive sensory analysis. J. Agric. Food Chem. 51:5067-5072.

Aston, J. W., and J. R. Dulley. 1982. Cheddar cheese flavour. Aust. J. Dairy Technol. 37:59-64.

Badings, H. T. 1991. Milk. Pages 91-106 in Volatile compounds in foods and beverages. H. Maarse, ed. Marcel Dekker, New York, NY.

Bendall, J. G. 2001. Aroma compounds of fresh milk from New Zealand cows fed different diets. J. Agric. Food Chem. 49:4825-4832.

Boscaini, E., S. V. Ruth, F. Biasioli, F. Gasperi, and M. D. Tilmann. 2003. Gas chromatography-olfactometry (GC-O) and proton transfer reaction-mass spectrometry (PTR-MS) analysis of the flavor profile of Grana Padano, Parmigiano Reggiano, and Grana Trentino cheeses. J. Agric. Food Chem. 51:1782-1790.

Counet, C., D. Callemien, C. Ouwerx, and S. Collin. 2002. Use of gas chromatography-olfactometry to identify key odorant compounds in dark chocolate. Comparison of samples before and after conching. J. Agric. Food Chem. 50:2385-2391.

Da Silva, M. A., V. Elder, C. L. Lederer, D. S. Lundahl, and M. R. McDaniel. 1993. Flavor properties and stability of a corn-based snack: relating sensory, gas chromatography, and mass spectrometry data. Pages 707-738 in Developments in Food Science. Vol. 
33. G. Charalambous, ed. Elsevier Publ., Amsterdam, The Netherlands.

Da Silva, M. A., D. S. Lundahl, and M. R. McDaniel. 1994. The capability and psychophysics of Osme: A new GC-olfactometry technique. Pages 191-209 in Developments in Food Science. Vol. 35. H. Maarse and D. G. van der Heij, ed. Elsevier Publ., Amsterdam, The Netherlands.

Ferretti, A., and V. P. Flanagan. 1971. Volatile constituents of whey powder subjected to accelerated browning. J. Dairy Sci. 54:1764-1768.

Friedman, M. 1996. Food browning and its prevention. J. Agric. Food Chem. 44:631-653.

Fu, S. G., Y. Yoon, and R. Bazemore. 2002. Aroma-active components in fermented bamboo shoots. J. Agric. Food Chem. 50:549-554.

Hugenholtz, J., M. Kleerebezem, M. Starrenburg, J. Delcour, W. de Vos, and P. Hols. 2000. Lactococcus lactis as a cell factory for high-level diacetyl production. Appl. Environ. Microbiol. 66:4112-4114.

Kaneko, T., H. Suzuki, and T. Takahashi. 1987. Influences of cellular components and redox potential of liquid concentrated culture of Lactobacillus bulgaricus on acid-producing activity and viability. J. Dairy Sci. 70:1128-1133.

Karagul-Yuceer, Y., K. R. Cadwallader, and M. A. Drake. 2003. Aroma-active components in liquid Cheddar whey. J. Food Sci. 68:1215-1219.

Karagul-Yuceer, Y., M. A. Drake, and K. R. Cadwallader. 2001. Aroma-active components of nonfat dry milk. J. Agric. Food Chem. 49:2948-2953.

Kieronczyk, A., S. Skeie, T. Langsrud, and M. Yvon. 2003. Cooperation between Lactococcus lactis and Nonstarter Lactobacilli in the formation of cheese aroma from amino acids. Appl. Environ. Microbiol. 69:734-739.

Kim, G. Y., J. H. Lee, and D. B. Min. 2003. Study of light-induced volatile compounds in goat's milk cheese. J. Agric. Food Chem. 51:1405-1409.

Livney, Y. D., and R. L. Bradley, Jr. 1994. Factors affecting flavor of whey as an ingredient in ice cream and other frozen desserts. J. Cult. Dairy Prod. 29:23-24, 26-27.

McDaniel, M. R., R. Miranda-Lopez, B. T. Watson, N. J. Michaels, and L. M. Libbey. 1990. Pinot noir aroma: A sensory/gas chromatographic approach. Pages 23-36 in flavor and off-flavors. Proceedings of the 6th International Flavor Conference, Rethymnon, Crete, Greece. G. Charalambous, ed. Elsevier Publ., Amsterdam, The Netherlands.

Miranda-Lopez, R., L. M. Libbey, B. T. Watson, and M. R. McDaniel. 1992. Odor analysis of Pinot noir wines from grapes of different maturities by a gas chromatography-olfactometry technique (Osme). J. Food Sci. 57:985-993, 1019.

Parlimnet, T. H., W. W. Nawar, and I. S. Fagerson. 1966. Origin of $\delta$-lactones in heated milk fat. J. Dairy Sci. 49:109-112.

Piggott, J. R. 1990. Relating sensory and chemical data to understand flavor. J. Sens. Stud. 4:261-272.

Qian, M., C. Nelson, and S. Bloomer. 2002. Evaluation of fat-derived aroma compounds in blue cheese by dynamic headspace GC/Olfactometry-Mass spectrometry. J. AOAC 79:663-667.

Qian, M., and G. Reineccius. 2002. Identification of aroma compounds in Parmigiano-Reggiano cheese by gas chromatography/olfactometry. J. Dairy Sci. 85:1362-1369.

Qian, M., and G. A. Reineccius. 2003. Quantification of aroma compounds in Parmigiano-Reggiano cheese by a dynamic headspace gas chromatography-mass spectrometry technique and calculation of odor activity value. J. Dairy Sci. 86:770-776.

Roberts, D. D., P. Polien, N. Antille, C. Lindinger, and C. Yeretzian. 2003. Comparison of nosespace, headspace, and sensory intensity ratings for the evaluation of flavor absorption by fat. J. Agric. Food Chem. 51:3636-3642.

Takahashi, K., M. Tadenuma, and S. Sato. 1976. 3-Hydroxy-4,5-dimethyl-2(5H)-furanone, a burnt flavouring compound from aged sake. Agric. Biol. Chem. 40:325-330.

Tomaino, R. T., J. D. Parker, and D. K. Larick. 2004. The effect of Lactococcus lactis starter cultures on the oxidative stability of liquid whey. J. Dairy Sci. 87:300-307.

USDA. 2004. U.S. Total whey powder production. United States Department of Agriculture. Online. Available: http://www.ams. usda.gov/dairy/mncs/graphs/whey_prod.pdf Accessed Mar. 15, 2004.

U.S. Dairy Export Council. 2004. Reference Manual for US Whey and Lactose Products. Online. Available: http://usdec.org/files/ pdfs/us08D 04.pdf. Accessed Sept. 23, 2004.

Van Den Dool, $\overline{\mathrm{H}}$., and P. Kratz. 1963. Generalization of the retention index system including linear temperature programmed gas liquid partition chromatography. J. Chromatogr. 11:463-471.

Wadodkar, U. R., J. S. Punjrath, and A. C. Shah. 2002. Evaluation of volatile compounds in different types of ghee using direct injection with gas chromatography-mass spectrometry. J. Dairy Res. 69:163-171.

Whetstine, C., J. D. Parker, M. A. Drake, and D. K. Larick. 2003. Determining flavor and flavor variability in commercially produced liquid Cheddar whey. J. Dairy Sci. 86:439-448.

Whitfield, F. B. 1992. Volatiles from interactions of Maillard reaction and lipids. Crit. Rev. Food Sci. Nut. 31:1-58. 